\title{
Summary of the HERA-LHC workshop
}

\author{
R. S. Thorne ${ }^{\mathrm{a}}$ \\ ${ }^{a}$ Department of Physics and Astronomy, University College London, Gower Street, London, WC1E \\ 6BT, United Kingdom
}

I present a summary of the last in the series of HERA-LHC workshops, CERN, 26-30th May 2008.

\section{Introduction}

In May 2008 the final stage of a 4-year series of meetings at DESY and CERN took place. The full timetable took the form

- 26-27 March 2005 CERN (250-300 participants)

- 6-9 June 2006 CERN (150 participants)

- 12-16 March 2007 DESY (160 participants)

- 26-30 May 2008 CERN (190 participants)

with a variety of additional smaller meetings, e.g. working weeks and working group meetings. The workshop was organised into 5 core working groups.

- Parton density functions

- Multi-jet final states and energy flows

- Heavy quarks (charm and beauty)

- Diffraction

- Monte Carlo tools.

I have been rather more involved with some of these groups than others, and am more qualified to talk on some than others. This summary will obviously reflect this, and will largely be highlights rather than a list. I will present the summary roughly by topic, but not always exactly by the Working Group, and indeed, the many overlapping sessions makes this impossible. I will also try not to dwell on topics covered in detail in other contributions to these proceedings.

\section{Parton distributions}

One of the main foci of the workshop was the issue of the extraction of the parton distributions, largely by using HERA data, and the consequences for LHC predictions. At this final meeting a number of new data sets were presented. The most interesting "new" data were clearly those on $F_{L}\left(x, Q^{2}\right)$, where it was shown that the earliest data [1] are consistent with NLO and NNLO predictions, but where the lower $Q^{2}$ data yet to be analysed could show some sensitivity to extended theoretical approaches. However, potentially the most important data presented (probably at the whole meeting) were the averaged HERA measurement of the total inclusive cross section [2. An example is shown in Fig. 1. As well as an improvement in statistics there is a significant elimination of correlated errors and the data has an accuracy of $1-2 \%$ over an enormous range of both $x$ and $Q^{2}$. When finally published it will be one of the best tests of factorization and perturbative QCD that we have. There is already a fit to these data performed by the experiments, with an impressive reduction in uncertainties [3. However, in comparing with other distributions there are lots of things to consider, e.g. different heavy flavour treatments, and particularly the number of free parameters used, which is small in these fits and likely to lead to constraints.

While waiting for these averaged data the PDFfitting groups provided presentations outlining many improvements and updates. There was a detailed discussion of the CTEQ6.6 PDFs [4, which contain a number of major changes. In par- 
Table 1

Ratios of predictions for $W \equiv W^{+}+W^{-}$and $Z$ total cross sections at the Tevatron $(\sqrt{s}=1.96 \mathrm{TeV})$ and LHC $(\sqrt{s}=14 \mathrm{TeV})$ calculated using the central values of different PDF sets, with respect to those from the MSTW 2008 sets.

\begin{tabular}{l|cc|cc}
\hline & \multicolumn{2}{|c|}{ Tevatron } & \multicolumn{2}{c}{ LHC } \\
Ratio to MSTW 2008 & $\sigma_{W}$ & $\sigma_{Z}$ & $\sigma_{W}$ & $\sigma_{Z}$ \\
\hline MRST 2006 NNLO & 1.00 & 1.01 & 0.99 & 1.00 \\
MRST 2004 NNLO & 0.99 & 1.00 & 0.93 & 0.94 \\
\hline MRST 2006 NLO (unpublished) & 0.99 & 1.00 & 1.00 & 1.01 \\
MRST 2004 NLO & 0.99 & 1.00 & 0.97 & 0.98 \\
CTEQ6.6 NLO & 0.98 & 0.99 & 1.02 & 1.02 \\
\hline
\end{tabular}

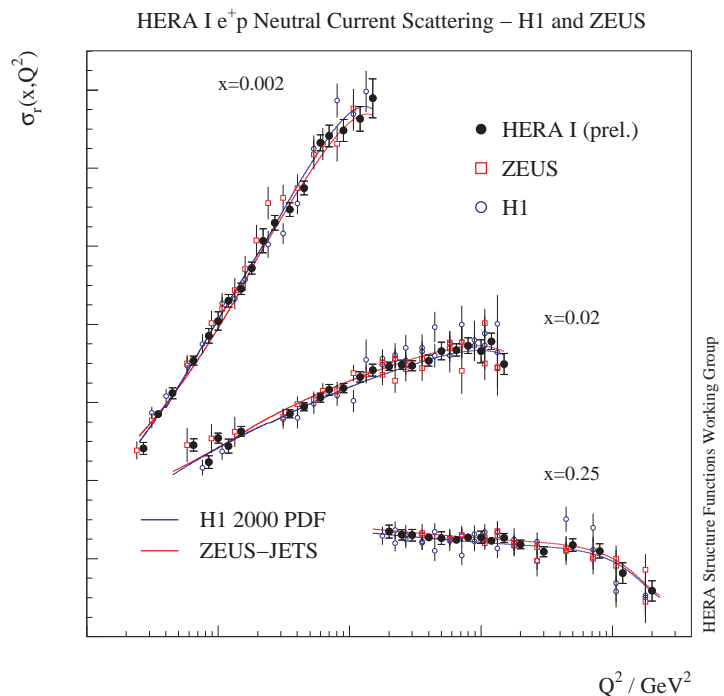

Figure 1. The averaged HERA structure function data in a sample of $x$ bins.

ticular it adopts the general-mass heavy flavour scheme as default (as does CTEQ6.5 [5]) and fits directly to strange quarks. There are now 44 (previously 40) eigenvector sets for the PDFs with uncertainties. The MSTW collaboration also presented a preliminary 2008 set [6] based on a fit to a very wide variety of new data. They now also fit

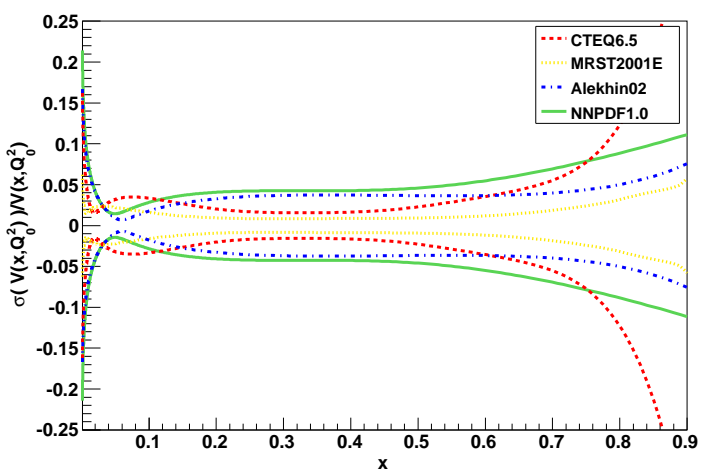

Figure 2. The NNPDF1.0 valence quark uncertainty compared to other sets.

the strange quark distribution directly but in this case it is much closer to the previous distribution, though the extra free parameters increase the uncertainties on all the light sea quarks. They now produce 40 (previously 30 ) eigenvector sets. The main MSTW focus was on a new dynamical determination of the tolerance, i.e. the $\Delta \chi^{2}$ used to determine the uncertainty. There is no longer a fixed value, but a systematic analysis eigenvector by eigenvector. They also highlighted the wide variety of new data included in the fit. Most important is the inclusion of new Tevatron data. This gives detailed information on quark decom- 
position, since it probes different weightings from the structure functions - for example, $d_{V}\left(x, Q^{2}\right)$ is now a different type of shape. Additionally the CDF and D0 Run II inclusive jet data in different rapidity bins 789 is fit very well and MSTW find a much softer high- $x$ gluon with these new data. One of the main consequences of these improvements in both analyses is a convergence of the predictions for cross sections at the LHC, as shown in Table 1

There was also the presentation of an entirely new set of PDFs by the NNPDF group [10, obtained by fitting to DIS data. This relaxes the restriction in uncertainty due to fixed parameterizations, though in practice relies on a very large number of parameters. It also proceeds by making a Monte Carlo sample of the distribution of experimental data by generating a large number of replicas of data centred on each data point with full inclusion of the information from errors and their correlations. Each replica is used to generate a PDF set, and the mean for a quantity is obtained by averaging, and the uncertainties from standard deviations. The data are split into training and validation sets for each replica, and the $\chi^{2}$ for one monitored while the other is minimised. This avoids over-complicating the input PDFs by stopping when the fit to the validation sets stops improving. The final fit quality seems slightly worse than in the global fits. The PDFs obtained seem to be fairly similar to the existing global fit sets (note at present they use a ZMVFNS and more limited quark decomposition). The uncertainties for valence quarks are shown in Fig. 2. In the region where the data are most constraining the uncertainty is quite similar to other approaches, but does tend to expand beyond this in regions where extrapolation is required, e.g. very high and low $x$. It must be remembered that there is less constraint from data than for CTEQ and MSTW at present. It will be interesting to see future developments.

\section{Standard candles}

The workshop included a special section on this topic. One of the central features was a presentation by representatives of each of ATLAS, CMS and $\mathrm{LHCb}$ on luminosity measurements. A detailed breakdown of expectations for LHCb can be seen in Table 2 11. To summarise, at LHCb, ATLAS and CMS, in very early running total $Z$ boson production is probably the best determination since the theoretical uncertainty on the cross section including all uncertainty sources is $4-5 \%$. Later a measurement of $p p \rightarrow p p+\mu^{+}+\mu^{-}$ may provide the best long-term determination at LHCb, possibly $\sim 2 \%$, but anything comparable at ATLAS and CMS will require total and elastic cross section measurements using forward detectors, e.g. ALFA (ATLAS) and TOTEM (CMS), and dedicated short low luminosity runs using particular beam optics. This could achieve a luminosity determination of accuracy $3-5 \%$ after one year.

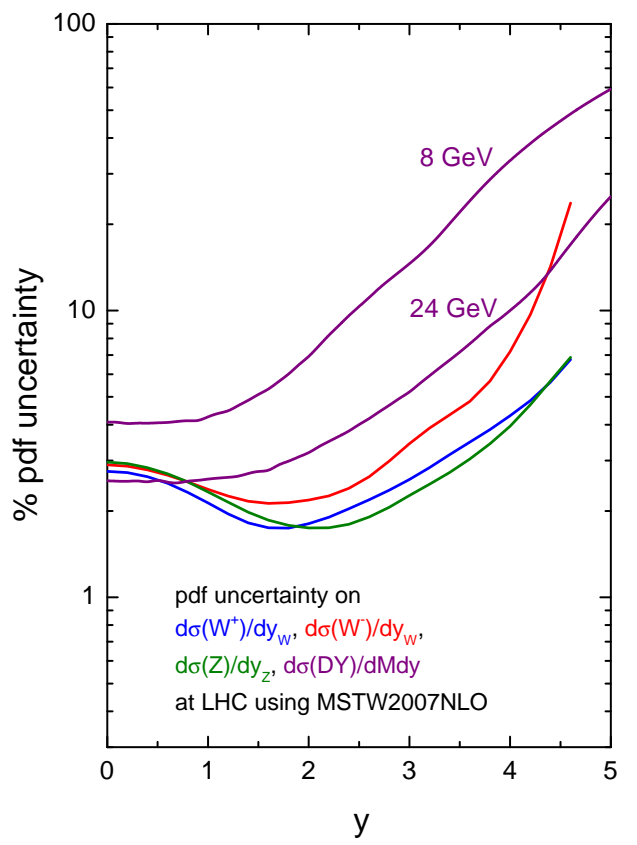

Figure 3. The uncertainty from PDFS on vector boson production.

It was also suggested by the CTEQ collaboration [4] that the total $t \bar{t}$ cross section could be 
Table 2

A summary of the potential for luminosity measurements at LHCb using various measurements and techniques.

\begin{tabular}{l|c|c|c}
\hline & $2008\left(5 \mathrm{pb}^{-1}\right)$ & $2009\left(0.5 \mathrm{fb}^{-1}\right)$ & $2010\left(2 \mathrm{fb}^{-1}\right)$ \\
\hline Van Der Meer & $20 \%$ & $5-10 \%$ & $5-10 \%$ \\
Beam Gas & $10 \%$ & $5<\%$ & $5<\%$ \\
$Z \rightarrow \mu^{+} \mu^{-}$ & $5 \%$ & $4 \%$ & $4 \%$ \\
$p p \rightarrow p p+\mu^{+}+\mu^{-}$ & $20 \%$ & $2.5 \%$ & $1.5 \%$ \\
\hline
\end{tabular}

a useful standard candle, with both theory and data uncertainties approaching $5 \%$. However, a presentation by Mangano [12] was in contradiction to this. The most-up-to-date predictions using NLO plus next-to-leading threshold logarithms for the $t \bar{t}$ cross section at $14 \mathrm{TeV}$ at the LHC for $m_{t}=171 \mathrm{GeV}$ were shown to be

$\sigma_{t \bar{t}}=908_{-85(9.3 \%)}^{+82(9.0 \%)}$ (scales) ${ }_{-29(3.2 \%)}^{+30(3.3 \%)}$ (PDFs) pb using CTEQ6.5 PDFs [5] and

$\sigma_{t \bar{t}}=961_{-91(9.4 \%)}^{+89(9.2 \%)}\left(\right.$ scales ${ }_{-12(1.2 \%)}^{+11(1.1 \%)}$ (PDFs) $\mathrm{pb}$ using MRST2006 PDFs 13. Hence, the scale uncertainty of order $9 \%$. Addtionally, although the PDF uncertainty using each set is much smaller, the central values differ by $\sim 6 \%$, due to systematic differences in the groups parameterisations and heavy flavour schemes. The effect from the latter may well be smaller with updated sets. Doubt was also expressed about the accuracy of the measurement reaching $5 \%$ for some time, but in the long term $t \bar{t}$ production may help constrain the gluon for $0.01<x<0.1$.

In the short term the best constraint on PDFs and the most precise comparisons of data and theory will be for electroweak boson production. The uncertainty on various cross sections due to PDFs is shown as a function of rapidity in Fig. 3. The uncertainty on $\sigma(Z)$ and $\sigma\left(W^{+}\right)$grows at high rapidity, that on $\sigma\left(W^{-}\right)$grows more quickly at very high $y$ since it depends on the less wellknown down quark, and the uncertainty on $\sigma\left(\gamma^{\star}\right)$ is greatest as $y$ increases, since it depends on the poorly know (even after HERA) partons at very small $x<10^{-4}$ 14. Measurements of all these bosons (and their ratios) will be good enough in one detector or another to put new constraints on PDFs quite quickly. This has the potential to be most dramatic for high rapidity, low mass DrellYan production which is possible at LHCb [15]. However, it was highlighted at the meeting that fully reliable calculations of all of these processes require great care. For real precision account of electroweak corrections must be made [16]. Also, small- $x$ resummations could give effects of a few $\%$, or possibly more for low mass Drell Yan. There were presentations from the groups working in this field 17/18/19. Though the groups are at various stages of development, it seems clear there is broad agreement on qualitative features, but some variation in approaches which lead to differences in details of results. It was pointed out that the measurements of $F_{L}\left(x, Q^{2}\right)$ at smaller $Q^{2}$ than has yet been analysed could give some information on resummation effects compared to fixed order calculations. They could also provide information on the success of dipole models.

\section{Dipole models, saturation}

Dipole models overlap with small- $x$ resummation, including some of the same corrections beyond a fixed order calculation. They are based on a less complete framework than the collinear factorization approach, but can be used in a wider region since they apply at low $Q^{2}$. A certain amount of modelling is always needed and added sophistication is continually being made to calculations in this approach. There is a clear overlap with the Diffraction working group. However, in general the free parameters in a dipole model are determined by a fit to $F_{2}\left(x, Q^{2}\right)$ data and results compared to more exclusive processes. An exam- 


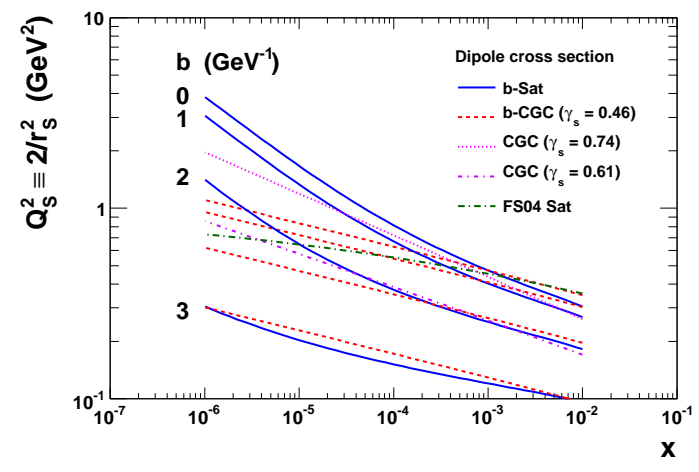

Figure 4. The saturation scale for a variety of dipole models.

ple of this was presented by Watt 20] using an extended dipole model with impact parameter dependence. This gives a good quality comparison to a wide variety of exclusive processes, though each of the two different models used works better in some cases. The saturation scale for this and a number of other approaches, all including heavy flavour contributions, is shown in Fig. 4 [21]. The saturation scale is seen to be at very low $x$ even for $b=0$, falling to lower $x$ as $b$ rises (the average for inclusive processes is $\left.b \sim 2-3 \mathrm{GeV}^{-1}\right)$. As seen, there are similar results from most other sophisticated and recent determinations of parameters using dipole models.

The issue of dipole models leads to geometric scaling. It has long been emphasised that to a reasonable approximation the HERA inclusive cross section can be described in terms of the variable $\tau=Q^{2}\left(x_{0} / x\right)^{\lambda}$ [22, though there are now variations of the precise functional form depending on the type of calculation. It has always been difficult to know how seriously to take this since it is broken (by higher orders in calculations, heavy quark contributions, ...) by significant amounts. In addition it was shown explicitly that geometric scaling can appear from standard DGLAP evolution 23. with sensible boundary conditions. Perhaps more striking was the

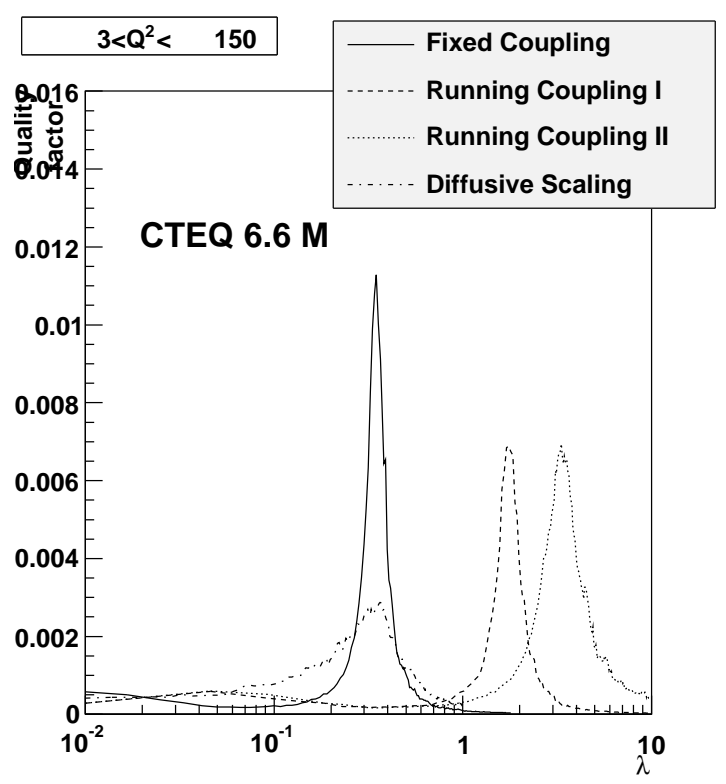

Figure 5. The quality factor for various types of geometric scaling using fixed order QCD and CTEQ PDFs.

observation that $F_{2}\left(x, Q^{2}\right)$ generated from MRST and CTEQ PDFs display all types of geometric scaling with good quality factors [24]. In this case there is no saturation in the generation at all, yet it is displayed by the theoretical output, as seen in Fig. 5, and indeed, more clearly than by real data. This, at the very least, suggests that geometric scaling is a general feature that is generated by saturation, but also in calculations where saturation is not used, or required.

\section{Perturbative QCD calculations}

There was a great deal of new activity in the field of jet definitions at the workshop. It has long been known that the initial cone-based jet algorithms are generally infrared unsafe. For example, in seeded cone algorithms the appearance of a single very soft particle can counterintuitively change the number of jets. In calculations this can lead to infrared sensitivity. As shown explic- 
itly by Salam, this has quantitative consequences in reality. Formally at some order a divergence will appear, i.e. we have a formal series of the form

$\alpha_{S}^{2}+\alpha_{S}^{3}+\alpha_{S}^{4} \times \infty$,

which in reality with some finite low scale cut-off $\Lambda$, becomes

$\alpha_{S}^{2}+\alpha_{S}^{3}+\alpha_{S}^{4} \times \ln \left(p_{t}^{2} / \Lambda^{2}\right)$,

and the incorrect last term is of size $\mathcal{O}\left(\alpha_{S}^{3}\right)$, so the result is meaningless beyond the lowest order. With different IR-unsafe algorithms and different quantities this breakdown of the expansion appears at different orders, sometimes at leading order.
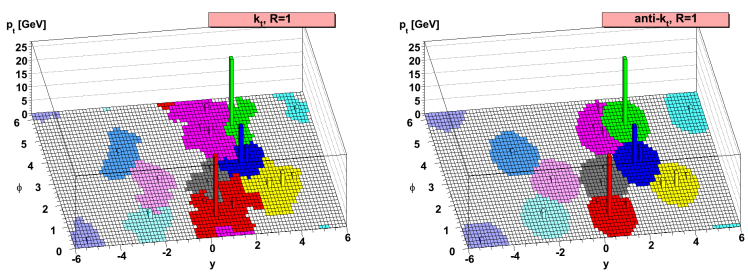

Figure 6 . Shape of jets using $k_{t}$ and anti- $k_{t}$ algorithms.

A new seedless infrared safe cone algorithm, SISCone 25] was presented. There was also the introduction of a "anti- $k_{t}$ algorithm" [26] which is a recombination algorithm based on the separation

$d_{i j}=\min \left(k_{t, i}^{-2}, k_{t, j}^{-2}\right)\left(\Delta \phi_{i j}^{2}+\Delta \eta_{i j}^{2}\right)$.

This has all the useful calculational properties of the IR-safe $k_{t}$ algorithm, but produces much more regularly shaped jets by combining all soft partons within a "cone" with a hard parton to produce a cone-like jet definition, as seen in Fig. 6. This is useful when using jet area to subtract underlying event and pile up, where $p_{T} / A$ is fairly constant except for hard jets [27.

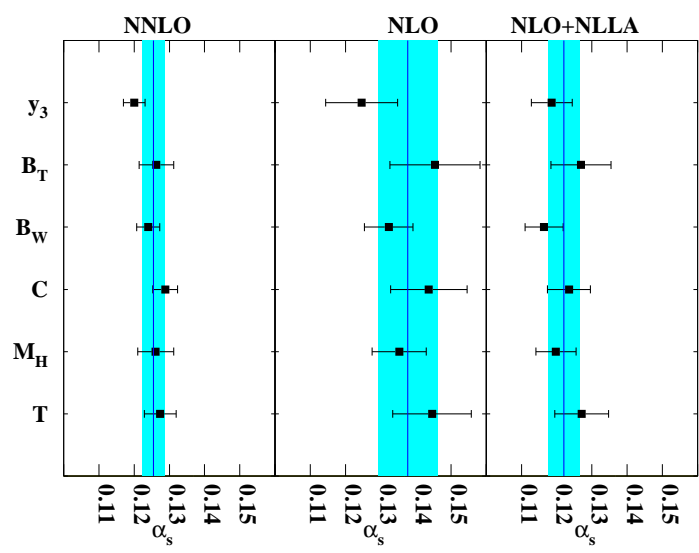

Figure 7. Determination of $\alpha_{S}\left(M_{Z}^{2}\right)$ from jet shapes.

Many new developments in the area of calculations in perturbative QCD were presented. These included automation of NLO calculations [28, improved parton showering which could be applied beyond LO [29], duality between one-loop and single-cut phase space integrals [30, automated one-loop $N$-gluon amplitudes via unitarity [31, automated implementation of dipole subtraction [32] and efficient ways of inputing NLO calculations into analyses 33134. There was also progress reported at NNLO. A full NNLO calculation of jet event shapes in $e^{+} e^{-}$annihilation was reported 35 as was the application to extracting $\alpha_{S}\left(M_{Z}^{2}\right)$ 36. The results can be seen in Fig. 7. The NNLO calculation reduces the uncertainty in the extraction of $\alpha_{S}\left(M_{Z}^{2}\right)$ compared to NLO, but leads to a high value. Combination with additional resummations is necessary and nearing completion. There was even major progress reported on the full NNLO calculation of heavy quark production in hadron-hadron collisions [37. The exact contributions for virtual corrections to quark annihilations have been calculated, and it was suggested that the total quark contributions may soon be known. 


\section{Heavy flavour}

One of the main features of the Heavy Flavours working group was a detailed comparison of variable flavour number schemes. The brief conclusion is that the MSTW and ACOT definitions of a General Mass Variable Flavour Number Scheme are very similar, but there are some outstanding differences in detail which can be thought of as higher order effects. A detailed summary can be found in 38. It was highlighted that when comparing with the precise new data up-to-date definitions and PDF sets should be used. There was some discussion on the present unavailability of fixed flavour number scheme calculations at NNLO, and preliminary steps in this direction were presented [39].

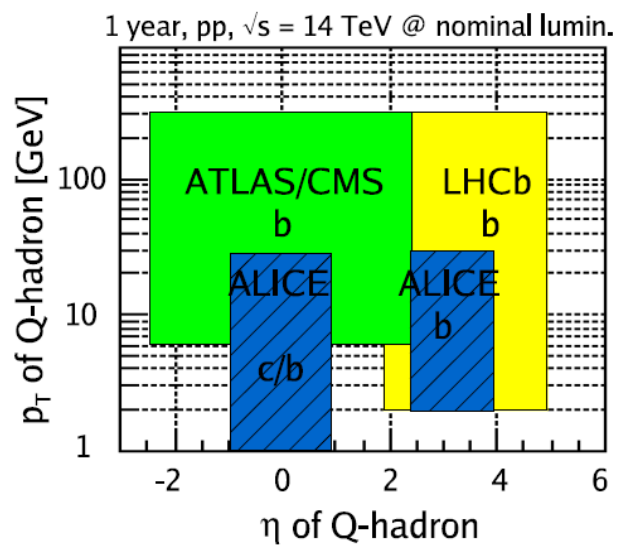

Figure 8. Acceptance for heavy flavours at the LHC.

There will be excellent coverage of heavy flavour at the LHC, as seen in Fig 8 and described in [0], with LHCb and particularly Alice having triggers to extremely low $p_{T}$. The enormous cross section for heavy flavour production at the LHC means it doesn't really matter that the bandwidth for the trigger for B physics is only about $5 \%$. All detectors have a wide rang- ing heavy flavour physics programme. Measurements of open heavy flavour production, heavy flavour jets, quarkonium production, oscillations of $B$ mesons and rare decays will all be covered by at least one of the experiments, and usually more. Measurements of heavy flavours down to low $p_{T}$ and at higher rapidity will test QCD in the same manner as the low-mass Drell-Yan production. This will constrain small- $x$ PDFs, check for small- $x$ resummations [4], saturation etc. However, theory is even more uncertain in this case. Predictions with largely similar underlying bases show uncertainties of factors of up to 2 40. Using more extreme theoretical approaches there is more scope for variations, so there is a lot of work for theorists here.

It was particularly noted that there is the possibility of very quick results on heavy meson production (Lytken). With $10 \mathrm{pb}^{-1}$ it will already be possible to measure $J / \psi$ polarisation to the same precision as the Tevatron with $1.3 \mathrm{fb}^{-1}$ but reaching to higher $p_{T}$. The same precision for $\Upsilon$ polarisation studies can be reached after 100 $\mathrm{pb}^{-1}$ and should help clarify the situation on the appropriate theoretical framework, with no clearcut result being available using present data 42 .

\section{Diffraction}

There have recently been major improvements in consistency between measurements, e.g. the comparison of the H1 [43] and ZEUS [44] inclusive diffractive cross sections using the Large Rapidity Gap definition in Fig. 9. In addition, inclusion of jet production has stabilised the results of the $\mathrm{H} 1$ fits dramatically [45, in fact bringing them near to the result in the alternative MRW approach [46. The level of agreement between experiments is such that it would now be appropriate to produce averaged data, and (subsequently) combined fits. The results of these could then be used at the LHC.

However, while diffractive PDFs obey factorization, i.e. one can determine evolution and combine with hard coefficient functions, the factorization is not universal. Very simple application of extracted PDFs to Tevatron data does not, and was never expected to work, giving results $\sim 10$ 


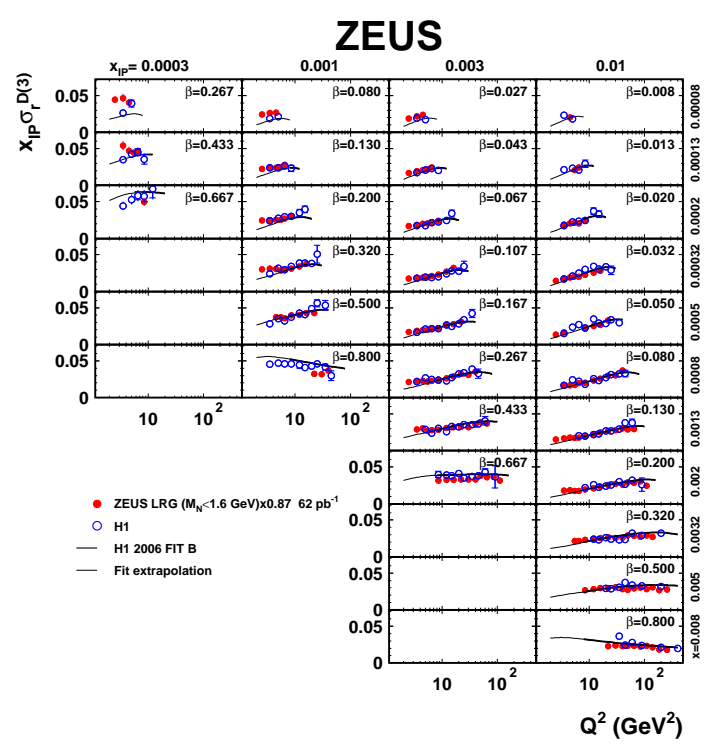

Figure 9. The large rapidity gap diffractive data from $\mathrm{H} 1$ and ZEUS.

times too high [47. Factorization is known to be broken in hadronic diffraction due to soft interaction filling in gaps in both initial and final states. This can be interpreted as a phenomenological "gap survival" probability. It is hoped this approach can give some reasonable accuracy for prediction of LHC processes, though the survival probabilities for different processes are not all alike. There is dependence on the nature of the basic process, kinematical configuration, cuts etc. 50. Predictions for diffractive processes at LHC can be tested with measurements at Tevatron [48, and evidence for doubly diffractive dijet production is seen in Fig. 10. The excess in dijet with a very large fraction of the total invariant mass of the final state is well described by the generator ExHuMe [49] based on the KMR calculations [50] with a $4.5 \%$ gap survival probability.

In principle there is a good test of factorisation also in diffractive photoproduction. The naive guess is that the direct contribution (like DIS) satisfies factorisation, but the resolved (like

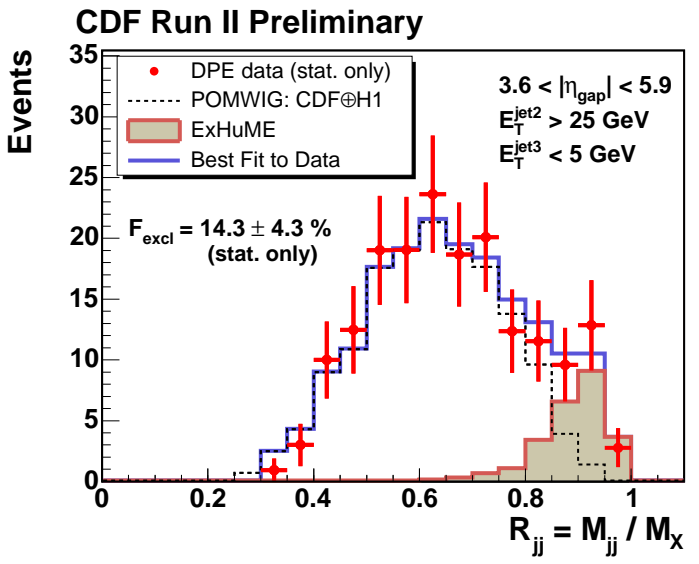

Figure 10. Dijet production at CDF.

hadronic processes) has a gap survival $\sim 0.3$. Initially ZEUS [51] and H1 data 52 did not agree well, and there was a suggestion of a suppression $\sim 0.5$ at all $x_{\gamma}$ in the latter but not the former. There have been recent improvements in understanding data differences with a suggestion of $E_{T}$ dependence, see e.g. [53]. It appears that suppression with a gap survival factor of $\sim 0.4$ independent of $x_{\gamma}$ works best, but this is still a matter of investigation [54.

\section{Monte Carlo, tools}

There have been a considerable number of improvements in Monte Carlo generators and associated tools during the time of the Workshop series, and many updates were noted. There were various developments in parton showers, e.g. 29. and [55. In particular a report on two new dipole showers ADICIC++ [56] and CSSHOWER++ [57] used in SHERPA was presented.

There was a comprehensive update report on the status of all the major Monte Carlo generators and associated tools. I begin with CASCADE [58. This is very different from standard Monte Carlo generators, and is based on generation of unintegrated PDFs via the CCFM equation. As such it has advantages, such as better treatment 


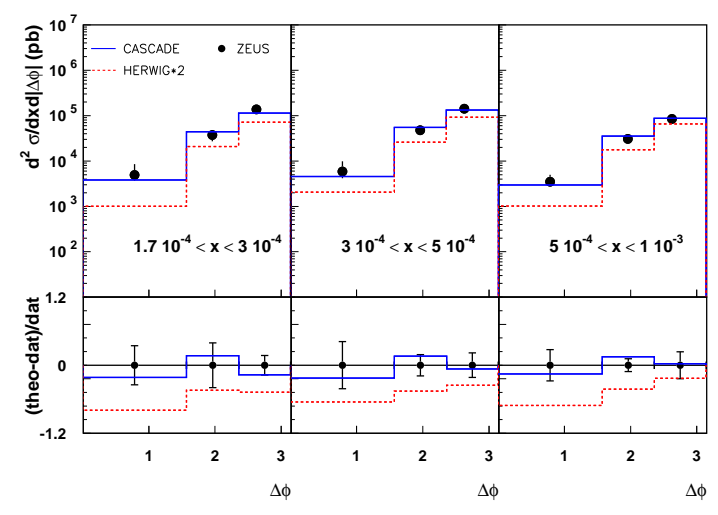

Figure 11. Angular correlations for 3-jet production comparing CASCADE and HERWIG++.

of high-energy logarithms, and is likely to be useful for processes sensitive to parton $k_{T}$. Successes for angular correlations in jets were outlined [59], and an example can be seen in Fig. 11. However, there are corresponding disadvantages, e.g. it is less complete in the high- $x$ regime, in particular currently not fully including quark contributions. Additionally knowledge of the unintegrated PDFs is required. All these may be important, and the most appropriate regime for the use of CASCADE should always be borne in mind, though improvements are continuing [60].

The ARIADNE generator 61] is based on a colour dipole cascade model. This has been completely rewritten in $\mathrm{C}++$ and has been validated for $e^{+} e^{-}$ annihilation, but some more work is needed for use at the LHC. As well as new parton showers, SHERPA 62 includes new cluster fragmentation 63] and a new method for calculating high multiplicity cross sections COMIX 64. Significant developments in the new update of HERWIG ++ 65] were reported, e.g. new models of decays, inclusion of more beyond the standard model physics (MSSM and extra dimension models), and inclusion of new hard processes. In particular a new model of multiple partonic interactions was presented [66], and a distinct improvement in the description of the underlying event

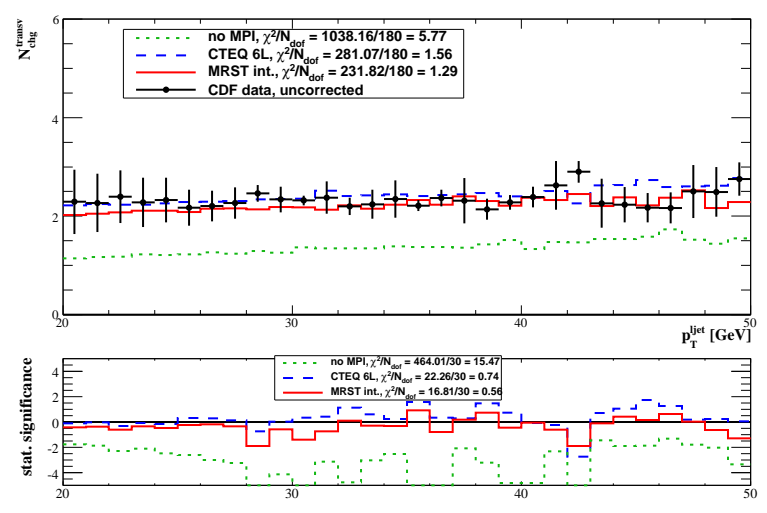

Figure 12. Predictions for number of charged particles using the multiparticle interaction model in HERWIG++.

at the Tevatron is seen, illustrated in Fig. 12 A summary of PYTHIA8 67] was reported, emphasising that it is a completely new generator. The improved features include interleaved $p_{T}$-ordered evolution, more underlying event processes, updated decay chains, the possibility for two selected hard interactions in the same event and the possibility to use one PDF sets for the hard process and another for the rest.

The last point is due to the suggestion that some PDFs, e.g. LO, are appropriate for some processes, e.g. underlying event, and others, e.g. NLO for others, e.g. $W, Z$ production [68. This brings me in a full circle back to PDFs. One of the issues discussed in detail in the series of workshops and in the offshoot PDF4LHC meetings has been the question of the PDFs to be used in LO Monte Carlo generators. The problems in using either standard LO or NLO PDFs in all cases was highlighted in 69. One alternative approach suggested by Jung is to obtain PDFs by fitting directly using Monte Carlo generators. Work on this project (PDF4MC) has begun using RAPGAP [70] to fit to HERA data on $F_{2}$, charm production and dijets. This is self-consistent, but will take a lot of time to do thoroughly and with anything like as wide constraints as the global fits, and a 

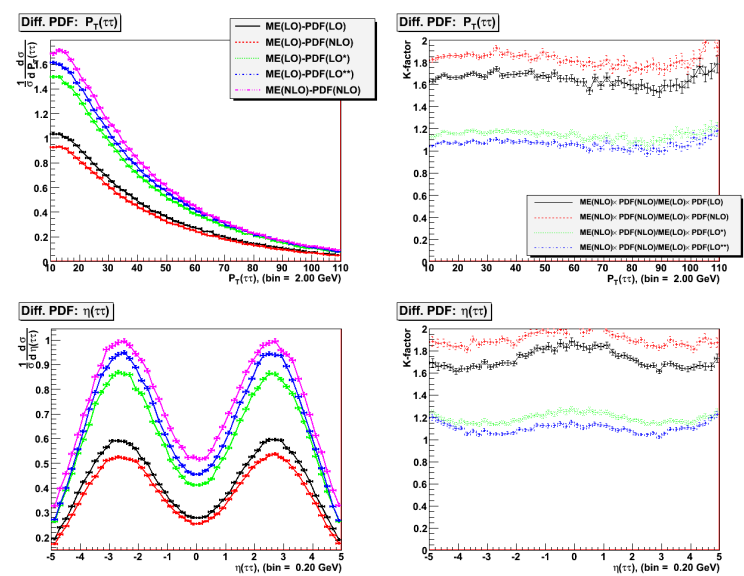

Figure 13. Predictions for $H \rightarrow \tau^{+} \tau^{-}$at full NLO and at LO with various choices of PDF.

good simultaneous fit to all data will be difficult to obtain. Indeed, the main problem with working entirely at LO is that most NLO corrections are positive, many large, rendering globally good fits and predictions impossible. This has led to the production of modified LO partons for LO Monte Carlo generators 69] where LO* PDFs are enhanced by allowing momentum violation and use of the NLO strong coupling. A further extension, $\mathrm{LO}^{* *}$, has a Monte Carlo inspired choice of scale in the coupling [71]. As seen in Fig. 13] these modified PDFs often lead to the best match to full NLO predictions. More generally, they provide results which are consistently very close to the full NLO result, so should be useful if a LO calculation is used due to time constraints or lack of a NLO generator. Similar modified LO sets are in preparation by CTEQ (presented at PDF4LHC in June 2008). These are also based (sometimes) on momentum violation in the input PDFs and sometimes in this case fitting to LHC pseudodata, possibly using $K$-factors for normalisations. This project of PDFs for use in Monte Carlos is one of many related to this series of workshops which is ongoing.

\section{Conclusions}

The objectives of the series of workshops, as defined by the organisers, were:

- To encourage and stimulate transfer of knowledge between the HERA and LHC communities and establish an ongoing interaction.

- To increase the quantitative understanding of the implications of HERA measurements for LHC physics.

- To encourage and stimulate theory and phenomenological efforts.

- To examine and improve theoretical and experimental tools.

- To identify and prioritise those measurements to be made at HERA which have an impact on the physics reach of the LHC.

The Workshop has clearly been very successful in all of these, and the organisers deserve congratulations and thanks. However, I have a couple of minor comments to add. As the summary shows, despite the name of the Workshop, Tevatron results and people have also made a big contribution and are involved in collaborations. Secondly, it was noticeable that quite a few people at the LHC who looked in at the first meeting decided to forget about importance of QCD. It is likely they will be obliged to remember when data starts to appear.

It is now the end of the HERA-LHC Workshop series in this form. However, the work started will undoubtedly carry on. The Parton Density Functions working group has developed into a PDF4LHC committee, and series of smaller workshops, which took place in February, July and September 2008, and will continue. There has already been a MC4LHC workshop, and there is continuing effort in the MCNET network. It would seem as though there is definitely scope for something similar along the lines of jets4LHC, diffraction4LHC, candb4LHC etc.. We really should build on the good work started and many collaborations established by the HERALHC Workshop series. 


\section{Acknowledgements}

I would like to thank Albert de Roeck and Hannes Jung for organising the HERA-LHC Workshop series so effectively, and the organisers of the Ringberg Workshop for inviting me to give this summary.

\section{REFERENCES}

1. F. D. Aaron et al. [H1 Collaboration], arXiv:0805.2809 [hep-ex].

2. H1 and ZEUS Collaboration, "Combination of $\mathrm{H} 1$ and ZEUS Deep Inelastic $e^{ \pm} p$ Scattering Cross Section measurements," submitted to The XXIII International Symposium on Lepton and Photon Interactions at High Energy, LP 2007, Aug. 13-18, Daegu,Korea.

3. B. C. Reisert [ZEUS Collaboration], arXiv:0809.4946 [hep-ex].

4. P. M. Nadolsky et al., Phys. Rev. D 78, 013004 (2008) [arXiv:0802.0007 [hep-ph]].

5. W. K. Tung, H. L. Lai, A. Belyaev, J. Pumplin, D. Stump and C. P. Yuan, JHEP 0702 (2007) 053 arXiv:hep-ph/0611254.

6. G. Watt, A. D. Martin, W. J. Stirling and R. S. Thorne, arXiv:0806.4890 [hep-ph].

7. A. Abulencia et al. [CDF - Run II Collaboration], Phys. Rev. D 75 (2007) 092006 [Erratum-ibid. D 75 (2007) 119901] arXiv:hep-ex/0701051.

8. V. M. Abazov et al. [D0 Collaboration], Phys. Rev. Lett. 101 (2008) 062001 arXiv:0802.2400 [hep-ex]].

9. T. Aaltonen et al. [CDF Collaboration], Phys. Rev. D 78 (2008) 052006 arXiv:0807.2204 [hep-ex]].

10. R. D. Ball et al. [NNPDF Collaboration], Nucl. Phys. B 809 (2009) 1 arXiv:0808.1231 [hep-ph]].

11. J. Anderson, M. Boonekamp, H. Burkhardt, M. Dittmar, V. Halyo, T. Petersen, "Experimental approaches: pp luminosity, standard candles, parton parton luminosity and PDFs at the LHC," to appear in the proceedings of the HERA-LHC meeting.

12. M. Cacciari, S. Frixione, M. L. Mangano, P. Nason and G. Ridolfi, JHEP 0809 (2008)
127 arXiv:0804.2800 [hep-ph]].

13. A. D. Martin, W. J. Stirling, R. S. Thorne and G. Watt, Phys. Lett. B 652 (2007) 292 arXiv:0706.0459 [hep-ph]].

14. R. S. Thorne, A. D. Martin, W. J. Stirling and G. Watt, arXiv:0808.1847 [hep-ph].

15. R. McNulty, arXiv:0810.2550 [hep-ex].

16. C. M. Carloni Calame, G. Montagna, O. Nicrosini and A. Vicini, JHEP 0612 (2006) 016 arXiv:hep-ph/0609170.

17. C. D. White and R. S. Thorne, Phys. Rev. D 75 (2007) 034005 arXiv:hep-ph/0611204.

18. G. Altarelli, R. D. Ball and S. Forte, Nucl. Phys. B 799 (2008) 199 arXiv:0802.0032 [hep-ph]].

19. M. Ciafaloni, D. Colferai, G. P. Salam and A. M. Stasto, JHEP 0708, 046 (2007) arXiv:0707.1453 [hep-ph]].

20. G. Watt and H. Kowalski, Phys. Rev. D 78 (2008) 014016 arXiv:0712.2670 [hep-ph]].

21. L. Motyka, K. Golec-Biernat and G. Watt, arXiv:0809.4191 [hep-ph].

22. A. M. Stasto, K. J. Golec-Biernat and J. Kwiecinski, Phys. Rev. Lett. 86 (2001) 596 arXiv:hep-ph/0007192.

23. F. Caola and S. Forte, Phys. Rev. Lett. 101 (2008) 022001 arXiv:0802.1878 [hep-ph]].

24. G. Beuf, C. Royon and D. Salek, arXiv:0810.5082 [hep-ph].

25. G. P. Salam and G. Soyez, JHEP 0705 (2007) 086 [arXiv:0704.0292 [hep-ph]].

26. M. Cacciari, G. P. Salam and G. Soyez, JHEP 0804 (2008) 063 arXiv:0802.1189 [hep-ph]].

27. M. Cacciari, G. P. Salam and G. Soyez, JHEP 0804 (2008) 005 arXiv:0802.1188 [hep-ph]].

28. A. van Hameren, J. Vollinga and S. Weinzierl, Eur. Phys. J. C 41 (2005) 361 arXiv:hep-ph/0502165.

29. Z. Nagy and D. E. Soper, JHEP 0807 (2008) 025 arXiv:0805.0216 [hep-ph]].

30. S. Catani, T. Gleisberg, F. Krauss, G. Rodrigo and J. C. Winter, JHEP 0809 (2008) 065 arXiv:0804.3170 [hep-ph]].

31. W. T. Giele and G. Zanderighi, arXiv:0805.2152 [hep-ph].

32. M. H. Seymour and C. Tevlin, arXiv:0803.2231 [hep-ph].

33. T. Carli, G. P. Salam and F. Siegert, 
arXiv:hep-ph/0510324, T. Carli, D. Clements et al., in preparation.

34. T. Kluge, K. Rabbertz and M. Wobisch, arXiv:hep-ph/0609285.

35. T. Gehrmann, G. Luisoni and H. Stenzel, Phys. Lett. B $\mathbf{6 6 4}$ (2008) 265 arXiv:0803.0695 [hep-ph]].

36. G. Dissertori, A. Gehrmann-De Ridder, T. Gehrmann, E. W. N. Glover, G. Heinrich and H. Stenzel, Nucl. Phys. Proc. Suppl. 183 (2008) 2 arXiv:0806.4601 [hep-ph].

37. M. Czakon, Phys. Lett. B 664 (2008) 307 arXiv:0803.1400 [hep-ph]].

38. R. S. Thorne and W. K. Tung, arXiv:0809.0714 [hep-ph].

39. I. Bierenbaum, J. Blumlein and S. Klein, arXiv:0806.0451 [hep-ph].

40. S. Alekhin et al., arXiv:hep-ph/0601013

41. R. D. Ball and R. K. Ellis, JHEP 0105 (2001) 053 arXiv:hep-ph/0101199.

42. V. M. Abazov et al. [D0 Collaboration], Phys. Rev. Lett. 101 (2008) 182004 arXiv:0804.2799 [hep-ex]].

43. A. Aktas et al. [H1 Collaboration], Eur. Phys. J. C 48 (2006) 715 arXiv:hep-ex/0606004.

44. M. Ruspa [ZEUS Collaboration], arXiv:0808.0833 [hep-ex].

45. A. Aktas et al. [H1 Collaboration], JHEP 0710 (2007) 042 arXiv:0708.3217 [hep-ex]].

46. A. D. Martin, M. G. Ryskin and G. Watt, Phys. Lett. B 644 (2007) 131 arXiv:hep-ph/0609273.

47. A. A. Affolder et al. [CDF Collaboration], Phys. Rev. Lett. 84 (2000) 5043.

48. T. Aaltonen et al. [CDF Collaboration], Phys. Rev. D 77 (2008) 052004 arXiv:0712.0604 [hep-ex]].

49. J. Monk and A. Pilkington, Comput. Phys. Commun. $175 \quad$ (2006) 232 arXiv:hep-ph/0502077.

50. V. A. Khoze, A. D. Martin and M. G. Ryskin, Eur. Phys. J. C 23 (2002) 311 arXiv:hep-ph/0111078.

51. S. Chekanov et al. [ZEUS Collaboration], Eur. Phys. J. C 52 (2007) 813 arXiv:0708.1415 [hep-ex]].

52. A. Aktas et al. [H1 Collaboration], Eur. Phys. J. C 51 (2007) 549 arXiv:hep-ex/0703022.
53. A. Schoning [H1 Collaboration], arXiv:0809.1050 [hep-ex].

54. M. Klasen and G. Kramer, Mod. Phys. Lett. A 23 (2008) 1885 arXiv:0806.2269 [hep-ph]].

55. S. Jadach, W. Placzek, M. Skrzypek and P. Stoklosa, arXiv:0812.3299 [hep-ph].

56. J. C. Winter and F. Krauss, JHEP 0807 (2008) 040 arXiv:0712.3913 [hep-ph]].

57. S. Schumann and F. Krauss, JHEP 0803 (2008) 038 arXiv:0709.1027 [hep-ph]].

58. H. Jung, Comput. Phys. Commun. 143 (2002) 100 arXiv:hep-ph/0109102.

59. F. Hautmann and H. Jung, JHEP 0810 (2008) 113 arXiv:0805.1049 [hep-ph]].

60. M. Deak, H. Jung and K. Kodak, arXiv:0807.2403 [hep-ph].

61. L. önblad, Comput. Phys. Commun. 71 (1992) 15.

62. T. Gleisberg, S. Hoche, F. Krauss, M. Schoenberg, S. Schumann, F. Siegert and J. Winter, arXiv:0811.4622 [hep-ph].

63. J. C. Winter, F. Krauss and G. Soff, $\begin{array}{llllll}\text { Eur. Phys. J. } & \text { C } & \mathbf{3 6} & \text { (2004) } & 381\end{array}$ arXiv:hep-ph/0311085.

64. T. Gleisberg and S. Hoche, JHEP 0812 (2008) 039 arXiv:0808.3674 [hep-ph]].

65. M. Bahr et al., Eur. Phys. J. C 58 (2008) 639 arXiv:0803.0883 [hep-ph]].

66. M. Bahr, S. Gieseke and M. H. Seymour, JHEP 0807 (2008) 076 arXiv:0803.3633 [hep-ph]].

67. T. Sjostrand, S. Mrenna and P. Skands, Comput. Phys. Commun. 178 (2008) 852 arXiv:0710.3820 [hep-ph]].

68. J. M. Campbell, J. W. Huston and W. J. Stirling, Rept. Prog. Phys. 70 (2007) 89 arXiv:hep-ph/0611148.

69. A. Sherstnev and R. S. Thorne, Eur. Phys. J. C 55 (2008) 553 arXiv:0711.2473 [hep-ph]].

70. H. Jung, Comput. Phys. Commun. 86 (1995) 147.

71. A. Sherstnev and R. S. Thorne, arXiv:0807.2132 [hep-ph]. 